\title{
Policy for developing clinical practice guidelines of Japanese Society for Dialysis Therapy
}

Kosaku Nitta ${ }^{1}$, Ikuto Masakane ${ }^{2,3,4^{*}}$, Tadashi Tomo ${ }^{2,3}$, Kenji Tsuchida ${ }^{2}$, Kiyoshi Ikeda ${ }^{3}$, Tomonari Ogawa ${ }^{3}$, Eiichiro Kanda ${ }^{3}$, Yoshihiko Kanno ${ }^{2,3}$, Yasuhiro Komatsu ${ }^{3}$, Masatomo Taniguchi ${ }^{3}$, Fumika Taki ${ }^{3}$, Takeshi Hasegawa ${ }^{3}$, Norio Hanafusa ${ }^{3}$, Takayuki Hamano ${ }^{3}$, Masafumi Fukagawa ${ }^{3}$, Jun Minakuchi ${ }^{3}$, Hiroyasu Yamamoto ${ }^{2,3}$, Naoki Washida ${ }^{3}$, Takashi Wada ${ }^{3}$, Yuzo Watanabe ${ }^{3}$ on behalf of the Scientific Academy Committee of Japanese Society for Dialysis Therapy (JSDT)

\begin{abstract}
Background: The Scientific Academy Committee of Japanese Society for Dialysis Therapy (JSDT) has developed 14 clinical practice guidelines (CPGs) and their revised editions independently or in cooperation with other organizations and translated many of them into English to make them available to the world. These guidelines were presented in a user-friendly textbook-like format and were useful for many healthcare professionals engaged in dialysis therapy. However, because the definition and the process for developing CPGs have recently become more rigorous worldwide, the conventional process of development and the format of JSDT no longer meet the requirements for $C P G s$.
\end{abstract}

Method: Since 2012, JSDT, with its Guideline Developing Working Group (formerly Guideline Subcommittee), had evaluated several CPGs developing systems. The working group evaluated the advantages and disadvantages of several CPGs developing systems. The most important point of them which JSDT would adopt for their own system was to be based on evidence and be able to receive international recognition.

Results: In Grading of Recommendations, Assessment, Development, and Evaluations (GRADE) system, the quality of evidence was evaluated by systematic review on each clinical outcome by systematic review panel. After the report from the systematic review panel, the CPG panel grades the strength of recommendation on each CPG statement. GRADE system was the most frequently used for developing CPGs by many human healthcare societies in the world. If JSDT adopt GRADE system, it is necessary for us to learn how to assess the quality of evidence through a systematic review, to develop a system for determining the strength of recommendation on the basis of a systematic review, and to decide how to manage any conflicts of interest.

Conclusions: JSDT should provide the useful information from Japanese experiences on chronic dialysis to the world. In order to achieve this aim, we concluded that the most reasonable approach is to utilize the data from JSDT Renal Data Registry in a more creative manner, publish such data for the world as evidence, and develop CPGs in accordance with a globally recognized methodology, GRADE.

(Continued on next page)

\footnotetext{
* Correspondence: imasakan.aipod@seieig.or.jp

${ }^{2}$ Scientific Academy Committee of Japanese Society for Dialysis Therapy,

Tokyo, Japan

${ }^{3}$ CPG Working Group, Scientific Academy Committee of Japanese Society for

Dialysis Therapy, Tokyo, Japan

Full list of author information is available at the end of the article
} provide a link to the Creative Commons license, and indicate if changes were made. The Creative Commons Public Domain Dedication waiver (http://creativecommons.org/publicdomain/zero/1.0/) applies to the data made available in this article, unless otherwise stated. 
(Continued from previous page)

Notification: The current manuscript is the position statement of JSDT for developing future CPGs and the second publication of "Policy for Developing Clinical Practice Guidelines (CPGs) of the Japanese Society for Dialysis Therapy" on J Jpn Soc Dial Ther 2016; 49: 453-62 (in Japanese), with permission.

Keywords: Clinical practice guideline (CPG), Systematic review (SR), Quality of evidence, Strength of recommendation, GRADE, Conflicts of interest (COI)

\section{Introduction}

Japanese Society for Dialysis Therapy (JSDT) has developed clinical practice guidelines (CPGs) for various fields of dialysis therapy to improve and standardize dialysis therapy in Japan. These CPGs have been providing useful information to many healthcare professionals engaged in dialysis therapy (Table 1). In addition, JSDT has made such information available to the world by publishing the English translation of those CPGs in Therapeutic Apheresis and Dialysis (TAD). The aims and the process of the development of JSDT CPGs were summarized in the committee report prepared by Fukagawa et al. in 2010 [1].

Recently, the requirements for CPGs have become more rigorous especially in transparency and fairness in the process of developing CPGs. Not only JSDT but all organizations that are engaged in the development of CPGs are faced with an urgent need to clarify their policy for developing their future CPGs. Since 2012, JSDT has discussed the basis of JSDT CPGs including the procedures for proposals for the development and revision of CPGs and the future direction of CPGs in the Guideline Subcommittee of the Scientific Academy Committee (now CPG Working Group). Because some CPGs were under development at that time, it took some time for making the committee consensus for the basis of JSDT CPGs. This position paper outlines the current global trend of CPGs, summarizes the characteristics and problems of past JSDT CPGs, and describes the policy for the development of future JSDT CPGs.

\section{Global trend toward CPG Definition of CPG}

The definition of CPGs by the Institute of Medicine (IOM) in the USA was "systematically developed statements to assist practitioner and patient decisions about

Table 1 JSDT CPGS

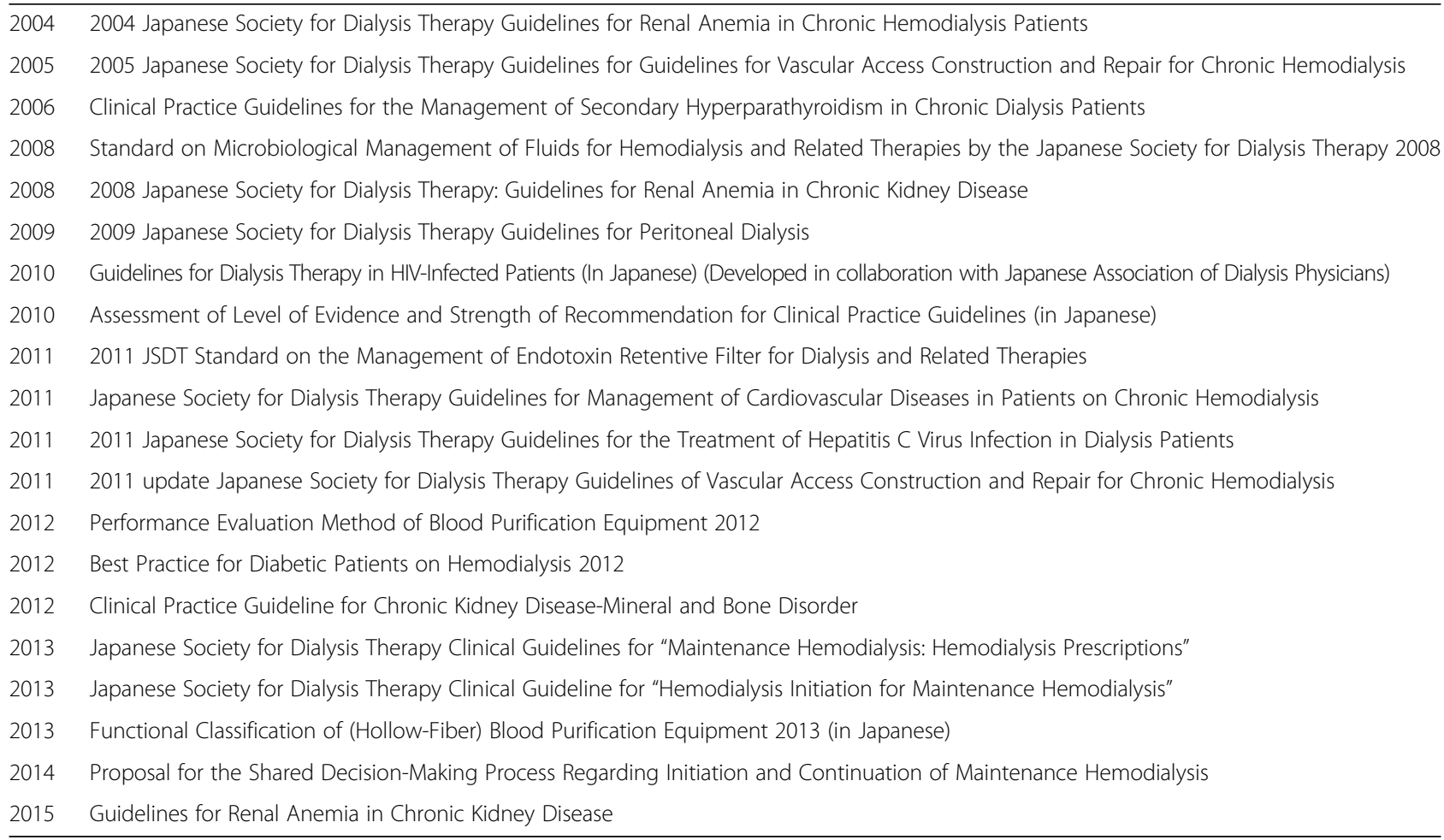


appropriate health care for specific clinical circumstances" in 1991 [2], but the definition was changed to "statements that include recommendations intended to optimize patient care that are informed by a systematic review of evidence and an assessment of the benefits and harms of alternative care options" in 2011 [3]. Both definitions agree in that CPGs assist to choose an appropriate treatment in clinical practice on the basis of unbiased information. However, a notable characteristic of the definition in 2011 is that it clarifies the process for developing CPGs by stating "that are informed by a systematic review of evidence and an assessment of the benefits and harms of alternative care options". In Japan, the development of CPGs is supported and assessed by the Japan Council for Quality Health Care as a part of the evidence-based medicine (EBM) project called the Medical Information Network Distribution System (Minds), which is sponsored by the Ministry of Health, Labor and Welfare [4]. Minds prepares the instructions for the development of CPGs [5, 6] and assesses the CPGs developed in Japan. Therefore, being included in Minds carries considerable prestige for CPGs developed in Japan. According to Minds 2014, a CPG is defined as "statements that present appropriate recommendations to assist patients and practitioners in making decisions regarding clinical practice of high importance, based on the body of evidence evaluated and integrated by systematic reviews and the balance between benefits and harms", which is almost the same as the definition provided by the IOM in 2011. In the USA, being "informed by a systematic review of evidence and an assessment of the benefits and harms of alternative care options" is a requirement to accept a CPG into the CPG list of National Guideline Clearinghouse (NGC) which plays a similar role as that of Minds in Japan [7]. It means that any CPGs without the above requirement should not be called "guideline" in the USA, a requirement which is now becoming a global trend.

Most academic societies in Japan, including JSDT, have developed CPGs, but processes for developing a CPG have not been standardized. Also, the terms "clinical practice guideline", "guide", or "clinical guide" have been simultaneously used without clear distinction from each other. If such "clinical practice guideline" or "clinical guide" is used just inside of Japanese, they are not exposed to international evaluation. However, when such guidelines are translated into English, they must be evaluated on the basis of whether the process of development meets the above requirement for CPGs. Therefore, whether the guidelines are published in English or not, it is necessary for academic societies to clarify their position on the strict definition of CPGs which is now globally accepted.

\section{Process for developing CPG}

Since the primary purpose of this report is to summarize the current problems of JSDT CPGs and the policy for the development of future CPGs, the details of the process of development are not exactly explained in this report. Alternatively, some key points in reviewing the CPGs developed in Japan are described on the basis of the Grading of Recommendations, Assessment, Development, and Evaluation (GRADE) system developed by the GRADE Working Group [8]. The GRADE Working Group, an informal collaboration group, was established in 2000 by Guyatt and Schunemann et al. who first used the term "EBM" formally in 1990 [9]. The GRADE system is now used worldwide by more than 90 academic societies and organizations, including WHO, as the international standard for the development of CPGs, and is also referred to by the NGC and Minds 2014, as mentioned above. Concerning the adoption of the GRADE system in Japan, Aihara discusses the details in the book "GRADE System for Clinical Practice Guidelines" [10], which is frequently referred to in this report.

First, the CPG development system should consist of two panels: (1) a panel that carries out a systematic review (SR) for clinical questions (CQs), assesses the quality of evidence, and prepares an evidence report (SR panel), and (2) a panel that determines the grade of recommendation for CPGs by examining the evidence report from various perspectives (CPG panel) (Fig. 1). The members of both panels may or may not overlap with each other, and these two groups work cooperatively. The SR panel is involved with purely academic considerations, while the role of the CPG panel is to assess the academic conclusions of the SR panel taking into account the actual state of society, from the perspectives of benefits and harms, medical economics, patients, and general people. In the selection of panel members, especially CPG panel members, it is important to manage conflicts of interest (COI) in accordance with predetermined rules. According to GRADE, it is necessary to set rules such that those who have a significant COI with the companies related to a CPG generally cannot assume key posts, nor do they have a right to vote in the CPG panel [10]. In 2007, Minds published "Minds Handbook for Clinical Practice Guideline Development 2007" [5], which served as the basis for the development of CPGs in Japan for a considerable time. This handbook, however, did not clarify the roles of the SR and the CGP panels or the rules for COI of panel members. The significance of improving the CPG development system was recognized in Japan only in 2010. 


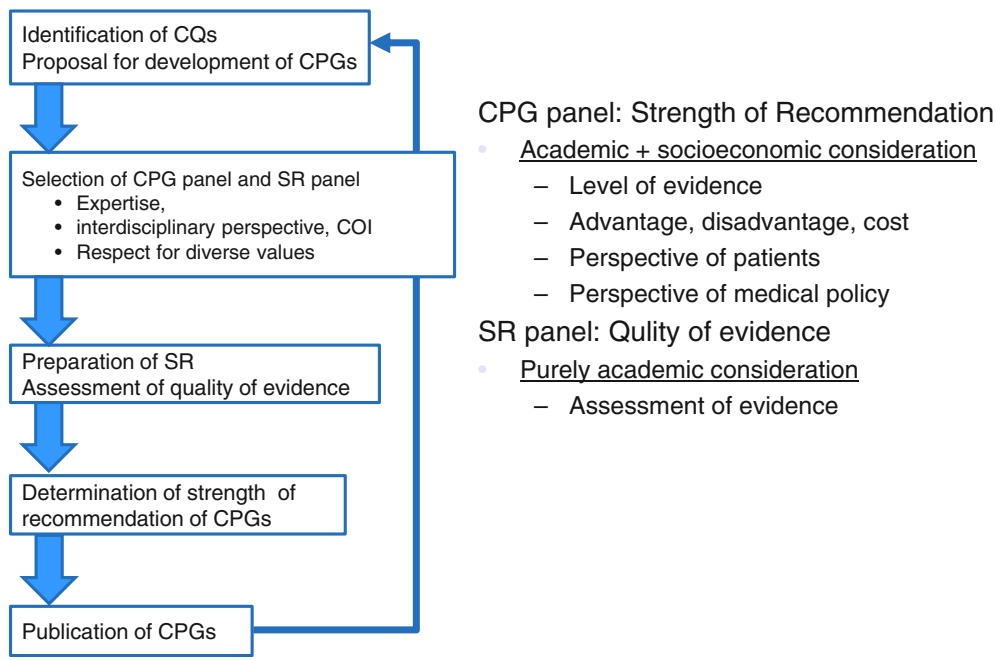

Fig. 1 Process of development of CPGs. COI conflict of interest, CQs clinical questions, CPG clinical practice guideline, SR systematic review. The scheme was composed by the author referenced on Ref [10] under approval of the author of Ref. [10]

\section{Characteristics and problems of JSDT CPGs Basic concept of JSDT CPGs}

The first JSDT CPG was the "Guidelines for Renal Anemia in Chronic Hemodialysis Patients" in 2004. The introduction of this CPG carried the following descriptions, which determined the direction of subsequent CPGs established by JSDT, and also clearly showed the characteristics of JSDT CPGs [11].

- Experts were called from related fields.

- While there are differences in the clinical practice patterns among Europe, the US, and Japan, little evidence has been accumulated in Japan.

- The data collected by JSDT renal data registry (JRDR) were treated importantly.

- The evidence obtained from foreign countries was re-evaluated in accordance to the clinical practices in Japan.

- The strength of the recommendation was discussed to achieve the committee consensus considering clinical practices in Japan.

- The aim of the CPGs is to provide guidance to JSDT members who are engaged in daily clinical practice.

This CPG, developed in 2004, consisted of a collection of expert opinions, including references to articles from other countries. Since it had not yet become common to create CPGs in response to CQs at that time, the CPGs were developed in a user-friendly textbook-like format.

Most of JSDT CPGs published have been translated into English. The committee report mentioned above explained the reason for English translation as follows: "The main purpose of JSDT CPGs is to improve the quality of life (QOL) of dialysis patients in Japan. However, as the most advanced country in Asia in terms of dialysis therapy, we also have a responsibility to provide useful information to the patients in Asian countries who are ethnically similar to Japanese" [1]. This statement suggested that the medical treatment required by patients in Europe and the USA might differ from that required by patients in Asia due to differences in diet, lifestyle, and comorbidities. Therefore, the data form JRDR was treated importantly to determine the quality of evidence for the development of CPGs. When such CPGs are translated into English and presented as "guidelines," they are subject to international assessment. Furthermore, if any country would adopt Japanese CPGs, they must want to know how they were developed and on what their recommendation strengths were decided. The European Renal Association/European Dialysis Transplantation Association (ERA/EDTA) clearly stated their position apart from the Kidney Disease/Improving Global Outcomes (KDIGO) guidelines, which are becoming a global standard for the treatment of kidney diseases. They changed the title of their recommendations from "guidelines" to "best practices" to incorporate region-specific factors for European countries into their statements [12]. However, because the "best practices" also indicate the recommendations with some strength, it is still necessary to clarify the process for determining the strength of recommendation.

\section{Background questions and foreground questions}

Both GRADE and Minds 2014 clearly stated that CPGs should be designed to address specific CQs. There are 
two types of questions arising from clinical practice; background questions and foreground questions, but that only foreground questions can be treated as CQs. Background questions are general questions about the pathology and treatment of a disease, such as, "What is renal anemia?" Background questions can be formulated into the following questions: "Who are the patients?", "What are the symptoms?", "When does it occur?", "Why does it occur?", and "How can it be treated?" Such information should be provided not by a CPG but by a textbook. On the other hand, foreground questions are questions about the selection of treatment and test methods in clinical practice, such as "Is treatment with "medicine A" recommended for this patient group?" Foreground questions are formulated into the following "Patient, intervention, comparison and outcome: PICO" questions and can be used as CQs that are to be addressed in CPGs: "for what kind of patients (patient)," "what kind of treatment (intervention)," "compared with what (comparison)," and "with what outcome (outcome)" [10]. Conventional JSDT CPGs were presented in a textbook-like format that was easy to use in clinical practice. A considerable part of the guidelines was devoted to answering background questions, which is inconsistent with the current definition of CPGs.

\section{Assessing the quality of evidence and grading the strength of recommendation}

The quality of evidence and the strength of recommendation for each CQ are very important because they are the main and most essential elements of a CPG. In JSDT CPGs, both the quality of evidence and the strength of recommendation have been determined on the basis of the committee report released in 2010 [1]. This committee report stated that "the evidence should be assessed in accordance with the KDIGO published in 2006," and Ref. 13 stated that KDIGO guidelines were developed in accordance with the methodology of GRADE [13]. The committee report released in 2010 [1] explained how the quality of evidence was assessed, stating, "In the assessment of various levels of evidence, we followed the assessment method presented by KDIGO in 2006, which was designed according to the methodology of GRADE. First, studies were classified as follows by their study design: randomized controlled trial (RCT) as high, observational study as low, and others as very low. Then, one or two points were added or deducted considering the content, quality, and bias of the evidence. Finally, the quality of evidence was determined as A (high), B (moderate), C (low), or D (very low)". This 4-scalegrading for the quality of evidence looks the same as the GRADE system [9] and then new Minds 2014 [6] at a glance. According to KDIGO, it was addressed that the quality of evidence should be assessed for each outcome.
However, the concept of the assessment of evidence for each outcome did not appear in the JSDT committee report, which resulted in the fixed idea that the quality of evidence was determined just for the study design of each article. The subsequent CPGs were developed with this misunderstanding. In Minds 2007 [5], it was stated that "the quality of evidence should be assessed for each question," which seemed to mean that the evidence should be assessed for each outcome. However, one may overlook this part without referring to the original text in GRADE because there was no description in Minds 2007 about the detailed assessment of the quality of evidence and the body of evidence for each outcome.

Bias in evidence must be assessed on the basis of a prescribed method [10]; however, there has been no detailed description on assessing the quality of evidence, such as the assessment for the quality of evidence of observational Japanese studies in each CPG. In SR, the method to assess the quality of evidence is prescribed, but the results may be affected by the skills and judgments of the individual reviewers who conduct the SR. This, however, does not mean that the reviewers can add or deduct points at their discretion. The reviewers should follow the prescribed method to ensure transparency and fairness.

The strength of recommendations of CPGs should be determined by the CPG panel, consisting of experts from related fields, clinicians, patients, and representative of citizens on the basis of the report submitted by the SR panel. The GRADE Working Group has proposed a methodology to derive an appropriate strength of recommendations, although the details are not provided here because they are beyond the scope of this report. There are two grades in the strength of recommendations, strong or weak. According to the committee report, in addition to these two strengths of recommendations, the opinions of the Scientific Academy Committee can be provided in JSDT CPGs, without indicating the level of evidence [1]. However, the GRADE Working Group has pointed out that GRADE does not accept any modification of the CPG developing process [10]

\section{Evaluation for JSDT CPGs by GRADE}

Dr. Aihara M, a member of the GRADE Working Group, has voluntarily assessed various CPGs in Japan related to GRADE and released the results on his website [14]. JSDT CPGs are subject to his assessment because JSDT states that JSDT CPGs applied GRADE system following KDIGO. As of September 2015, the CPGs for chronic kidney disease-mineral and bone disorder (CKD-MBD), vascular access, hepatitis $C$, and dialysis prescription have been evaluated and judged as "not meeting the criteria for using GRADE". This assessment is based on the eight 
minimum criteria for stating that GRADE is used [8, 10] (Table 2). In 2012 when JSDT recognized the need to fundamentally reform the process for developing CPGs, only three CPGs from the Japanese Society for Temporomandibular Joint had been developed in accordance with the GRADE methodology in Japan. As of January 2016, there are seven CPGs for five organizations [14].

\section{Policy for developing future JSDT CPGs CPG development system}

As previously described, the definition of CPGs has become more rigorous and is now globally accepted. Under such circumstances, JSDT also should reform the process for the development of CPGs. It is not realistic to create own approach for the development of future CPGs, but it is desirable to develop CPGs in accordance with a system which has been globally accepted and adopted by existing organizations. It is necessary to use a globally accepted system to continue to provide information to Asian countries, which has been one of the major purposes of JSDT CPGs. The CPG development systems and the CPG and SR libraries that are internationally recognized include the Cochrane Collaboration [15], the NGC [7], and the Centre for EvidenceBased Medicine (CEBM) [16], in addition to GRADE. Also in Japan [6], there is Minds 2014. The CPGs of The British Medical Journal (BMJ), the Center of Disease Control (CDC), UpToDate, and KDIGO have been

Table 2 Minimum criteria for stating "GRADE is used"

GRADE is an outcome-specific rating of confidence (a body of evidence, not an individual study).

"Quality of evidence" should be defined consistently with one of the two definitions (for guidelines or for systematic reviews) used by the GRADE Working Group.

Explicit consideration should be given to each of the GRADE criteria for assessing the quality of evidence.

The overall quality of evidence should be assessed for each important outcome and expressed using four categories ("high," "moderate," "low," and "very low").

Evidence summaries (narrative or tabular) should be used as the basis for judgments about the quality of evidence and the strength of recommendations.

Explicit consideration should be given to each of the GRADE criteria for assessing the strength of a recommendation.

The strength of recommendations should be expressed using two categories (weak/conditional and strong) for or against a management option, and the definitions for each category should be consistent with those used by the GRADE Working Group.

Decisions about the strength of the recommendations should ideally be transparently reported (e.g.. GRADE grid).

Each element was arranged expression from Ref. [8, 10]

Abbreviation: GRADE the Grading of Recommendations, Assessment,

Development, and Evaluation developed in accordance with GRADE [8], and the use of GRADE is also recommended in Minds 2014 [6]. In addition, a detailed commentary on GRADE has been published in Japanese by Aihara [10]. In the Cochrane Collaboration [17], the SRs principally do not provide any recommendations. Therefore, when we try to make a CPG using a SR from the Cochrane library, we should determine the strength of recommendation for each $\mathrm{CQ}$ based on the quality of evidence as well as the balance between the benefits and harms in the clinical and socio-economic environment in Japan. Also, at present, there are very few reviews on dialysis therapy in the Cochrane Library and are not frequently referred to. The requirements to be included in the NGC are based on the definition of CPGs provided by IOM in 2011; namely, the grade of recommendation must be determined after carrying out an SR. On the other hand, CEBM describes GRADE as a complicated, less effective, and time-consuming approach, and maintains its original methodology [16]. Upon comprehensive consideration of these issues, we concluded that it is most appropriate for JSDT to develop future CPGs in accordance with the GRADE system. Minds 2014 contains recommendations for the development of CPGs in Japan, but the system adopted by Minds 2014 is almost the same as that of GRADE, as a result of significant revision of Minds 2007. Also, Minds 2014 itself recommends the GRADE method [6]. When we develop CPGs in accordance with GRADE, they necessarily meet the requirements of Minds 2014. As a result, they will be regarded as reliable guidelines in Japan and, at the same time, can gain international recognition. Adopting GRADE means that CPGs will be developed in response to $\mathrm{CQs}$ arising from clinical practice and that the SR and CPG panels will be organized for the assessment of the quality of evidence and the determination of the strength of recommendation on the basis of the prescribed method.

\section{Proposals for development and revision}

Given the speed of recent medical advances, the lifetime of CPGs is generally said to be 4 years. However, it is both physically and economically impossible to revise all existing CPGs every 4 years. Also, as has been described, the existing JSDT CPGs contain a number of background questions and descriptions of general knowledge, which is not consistent with the current definition of CPGs. Therefore, it is important to define the future procedures regarding proposals for the development and revision of CPGs.

Because CPGs are developed in response to CQs, the CQs that should be addressed in CPGs are contentious and confusing issues in the current clinical practice of dialysis therapy. There are several considerations for 
identifying high-priority CQs. First, it is necessary to check if there are contradictions between the statements of existing CPGs and the latest medical knowledge gained in clinical practice. If there are contradictions, a literature review will be conducted to find the latest trends in testing and treatment. If it is necessary to grasp the current situation, this will be done by analyzing the existing data from statistical surveys or conducting other statistical surveys with new items. High-priority CQs that should be addressed in CPGs are identified through these processes. Also, the purpose of a literature review is not only the assessment of existing CPGs; the possibility of the development of CPGs based on the latest medical knowledge about what should be applied in clinical practice is also another purpose of a literature review. In 2015, the Scientific Academy Committee launched a new system named "Dialysis therapy, year in review" at the annual meeting. In this system, the subcommittee members of the Scientific Academy committee present a year-in-review in their area of concern in dialysis therapy [18]. Only literature reviews were presented in the first year, 2015. However, in the future, they will evaluate the existing CPGs and the need for CPGs which incorporate new findings and will propose the revision and development of CPGs to the Scientific Academy Committee. The Scientific Academy Committee will examine the necessity and the urgency of both the revision and development of CPGs proposed by the subcommittees in each area of concern and will decide which CPGs need to be developed. The development of new a CPG will start after being approved by the Executive Board (Table 3). The overall year-round schedule is as follows: Year-in-reviews will be presented at the annual meeting held in June; the proposals for the development and revision of CPGs will be submitted to the Scientific Academy Committee during the period from October to December; and the Scientific Academy Committee

Table 3 Process of proposals for development and revision of CPGs

1. Identification of urgent CQs (September)
Examination of validity of existing CPGs
New CQs from literature review
2. Proposals for development and revision of CPGs
(October-December)
3. Decision of development or revision of CPG (January)
Selection of candidate CQs
Assignment of CPG and SR panels (disclosure of COI)
4. Approval of project of development and revision of CPGs (March)
5. Start of development of CPGs (SR and CPG panels) (April)

Each month shown in the right edge means the ideal working period for efficiently developing CPG will decide which CPGs need to be developed or revised in January and obtain approval of the project by the Executive Board in March. Such a schedule would be effective in that it allows the project to be continued even when the election of board members takes place.

\section{Assignment of CPG and SR panel members and COI}

The Scientific Academy Committee will examine the need for the development and revision of CPGs and then submit the proposals for the development of CPGs to the Executive Board, clarifying its purpose and the outline of CQs, to obtain approval for the project. In the selection of CPG and SR panel members, the Scientific Academy Committee will disclose the COI of all candidates and draft a list of panel members considering the qualifications of candidates and the degree of any COI. The chair of the Scientific Academy Committee will submit the outline of CQs, the draft list of CPG and SR panel members, the COI information, and the reasons for recommendation of the candidates to the COI Committee. The COI Committee will review the draft of the list of CQs and SR panel members, as a third party, and notify the chair of the Scientific Academy Committee of the results. The chair of the Scientific Academy Committee will then submit the draft list of CPG and SR panel members which has been reviewed by the COI Committee to the Executive Board for deliberation. In accordance with GRADE, the CPG panel will consist of representatives from various fields, including representatives of patients. Details of the members will be specified according to the nature of each CPG. The addition of new members to each panel is allowed in principle, but the process described above must be followed in the selection of new members. The COI of panel members will be disclosed to the public at the time of solicitation of public comment. The COI will be disclosed in the format prescribed by JSDT. It is necessary for JSDT to further discuss the management of COI in the development of JSDT CPGs, considering the social changes surrounding medical practice and the opinions of the Japanese Association of Medical Sciences.

\section{Quality of evidence and strength of recommendation}

Both the quality of evidence and the strength of recommendation in CPG statements should be determined in accordance with the GRADE system. The quality of evidence will be classified into four levels: high, moderate, low, and very low. The grade of recommendation will be classified as strong or weak $[10,19]$ (Tables 4 and 5). 
Table 4 Levels of quality of evidence and their definition in GRADE approach

\begin{tabular}{lll}
\hline Quality of evidence & Conventional definition & New definition \\
\hline High & $\begin{array}{l}\text { Further research is very unlikely to change our confidence in the } \\
\text { estimated effect. }\end{array}$ & $\begin{array}{l}\text { We are very confident that the true effect lies close to that } \\
\text { of the estimated effect. }\end{array}$ \\
Moderate & $\begin{array}{l}\text { Further research is likely to have an important impact on our } \\
\text { confidence in the estimated effect and may change the estimate. }\end{array}$ & $\begin{array}{l}\text { We are moderately confident in the effect estimate: The true } \\
\text { effect is likely to be close to the estimated effect, but there } \\
\text { is a possibility that it is substantially different. }\end{array}$ \\
Low & $\begin{array}{l}\text { Further research is very likely to have an important impact on our } \\
\text { confidence in the estimated effect and is likely to change the } \\
\text { estimate }\end{array}$ & $\begin{array}{l}\text { Our confidence in the effect estimate is limited: The true } \\
\text { effect may be substantially different from the estimated effect. }\end{array}$ \\
Very low & Any estimate of effect is very uncertain. & $\begin{array}{l}\text { We have very little confidence in the estimate: The true effect } \\
\text { is likely to be substantially different from the estimated effect. }\end{array}$
\end{tabular}

The table was drawn from Ref. [10] but the definitions were originally from Ref [19]

\section{Process flow for publication of CPGs}

The chair of the CPG panel will submit the working draft (WD) consisting of CPG statements, strength of recommendation, and SR report to the chair of the Scientific Academy Committee. The chair of the Scientific Academy Committee will prepare the committee draft (CD) after having discussion and achieving consensus with the CPG panel about the grade of recommendation in the WD. Then, the chair of the Academic Committee will call a consensus conference of JSDT members at the annual meeting or at another time to summarize the opinions of JSDT members. The CPG panel will prepare the draft for CPG (D-CPG) and submit it to the Executive Board through the chair of the Academic Committee. On the basis of the D-CPG, the final draft for CPG (FDCPG) will be prepared incorporating the collective opinion of the Executive Board. The FD-CPG will be posted on the JSDT website, followed by the solicitation of public comment and a public hearing. The final version of CPGs will be developed incorporating both public comment and the opinions presented in the public hearing, which will be published upon approval of the Executive Board (Table 6).

\section{System for creating evidence}

The author of this report has participated in the development of a number of JSDT CPGs and has often heard someone remark during a conference, "There are few evidences in this field." However, evidence is not necessarily limited to an RCT. Currently, a method is being formulated to assess the quality of evidence provided by observational study. In addition, new epidemiological analysis methods are being devised so that a large-scale epidemiological study can also provide evidence which is comparable to an RCT. Furthermore, the cohort data of

Table $\mathbf{5}$ Implications of strength of recommendation for different users of guidelines

\begin{tabular}{lll}
\hline Grade of recommendation & Strong & Weak \\
\hline Definition & $\begin{array}{l}\text { A guideline panel is confident that the desirable } \\
\text { effects (benefits) of an intervention outweigh } \\
\text { undesirable effects (harms, burden, and costs), } \\
\text { or vice versa. }\end{array}$ & $\begin{array}{l}\text { Desirable effects (benefits) of an intervention probably outweigh } \\
\text { the undesirable effects (harms, burden, and costs), or vice versa, } \\
\text { but the panel is less confident. }\end{array}$ \\
$\begin{array}{lll}\text { Fost individuals in this situation would want the } \\
\text { recommended course of action, and only a small } \\
\text { proportion would not. }\end{array}$ & $\begin{array}{l}\text { Many individuals in this situation may or may want the } \\
\text { suggested course of action. }\end{array}$ \\
$\begin{array}{lll}\text { Most individuals should receive the intervention. } \\
\text { Adherence to this recommendation according to } \\
\text { the guidelines could be used as a quality criterion } \\
\text { or performance indicator. Formal decision-making } \\
\text { aids are not likely to be needed to help individuals } \\
\text { make decisions consistent with their values and } \\
\text { preferences. }\end{array}$ & $\begin{array}{l}\text { Recognize that different choices will be appropriate for different } \\
\text { patients, and that you must help each patient arrive at a } \\
\text { management decision consistent with her or his values and } \\
\text { preferences. Decision-making aids may well be useful in helping } \\
\text { individuals to make decisions consistent with their values and } \\
\text { preferences. Clinicians should expect to spend more time with } \\
\text { patients when working toward a decision. }\end{array}$ \\
$\begin{array}{lll}\text { The recommendation can be adapted as a policy } \\
\text { in most situations, including for the use as } \\
\text { performance indicators. }\end{array}$ & $\begin{array}{l}\text { Policy-making will require substantial debates and involvement } \\
\text { of many stakeholders. Performance indicators would have to } \\
\text { focus on the fact that adequate deliberation about the } \\
\text { management options has taken place. }\end{array}$ \\
\hline
\end{tabular}


Table 6 Flow chart from development to publication of CPGs

\begin{tabular}{l} 
Working draft (WD) \\
WD is submitted by CPG panel to Chair of Academic Committee \\
Including CPG statements, grade of recommendation, SR report providing \\
basis of statements and grade of recommendation, and other materials \\
Committee draft (CD) \\
CD is after deliberation on WD by Academic Committee \\
Prepared through consensus conference of CPG panel and Academic \\
Committee \\
Draft for CPG (D-CPG) \\
D-CPG is developed through consensus conference of JSDT members \\
on CD \\
Final draft for CPG (FD-CPG) \\
FD-CPG is prepared through consensus conference of Executive \\
Board on D-CPG \\
Posted on JSDT website, followed by solicitation of public comments \\
and public hearing \\
Preparation of CPG \\
Prepared incorporating public comments and opinions presented in \\
public hearing \\
Published upon approval of Executive Board \\
Publication of CPG \\
Published in journals in Japanese and English \\
\hline
\end{tabular}

750,000 patients have been obtained by JSDT Renal Data registry. In order to fully utilize such data as a source of evidence in the development of future CPGs, it is necessary to make it available in the form of articles published in English. The desirable cycle of the development of
CPGs is as follows: existing CPGs are assessed in terms of CQs raised in clinical practice and on the basis of literature reviews, necessary data are analyzed and published in the form of articles, CPGs are developed, the CPGs are reflected in medical policy and in the revision of medical fees, and the effects and validity of the CPGs are reexamined (Fig. 2). It is expected that JSDT will facilitate this cycle in the future. As mentioned previously, the Scientific Academy Committee and the JRDR Committee have already launched such a system [18].

\section{Application of CPGs in clinical practice}

As described previously, existing JSDT CPGs covered all areas of interest in chronic dialysis therapy and all topics in each area so that even beginners could understand the direction of clinical practice upon first reading (Fig. 3). As a result, the CPGs were presented in a userfriendly textbook-like format and were useful for many healthcare professionals but were not consistent with the current definition of CPGs. In the future, not only JSDT but all academic societies must develop CPGs in the form of answers to CQs. Clinical practice, however, does not necessarily proceed in the manner of CQs. Therefore, while CPGs should be developed in accordance with the officially accepted methodology, they will become more useful in daily clinical practice if there is a comprehensive document explaining the situations in clinical practice to which those CPGs apply. It is desirable that the significance of each CPG relative to those of other CPGs is indicated by a textbook-like document covering the entire area of concern in dialysis therapy

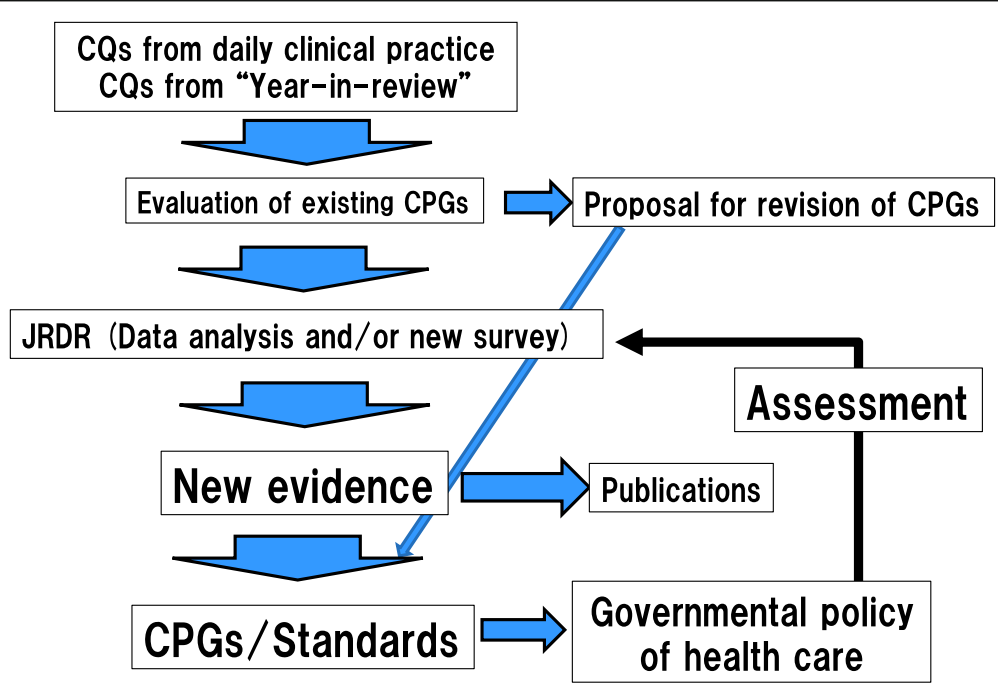

Fig. 2 New academic flow chart of JSDT. JSDT the Japanese Society for Dialysis Therapy, CQs clinical questions, CPGs clinical guidelines, JRDR JSDT Renal Data Registry. In order for JSDT and other academic societies to develop CPGs of high quality, it is necessary to efficiently facilitate the cycle of setting CQs, conducting surveys, creating evidence, developing CPGs, reflecting CPGs in health service, and asessing the effects. The figure was quoted from Ref. [18] 


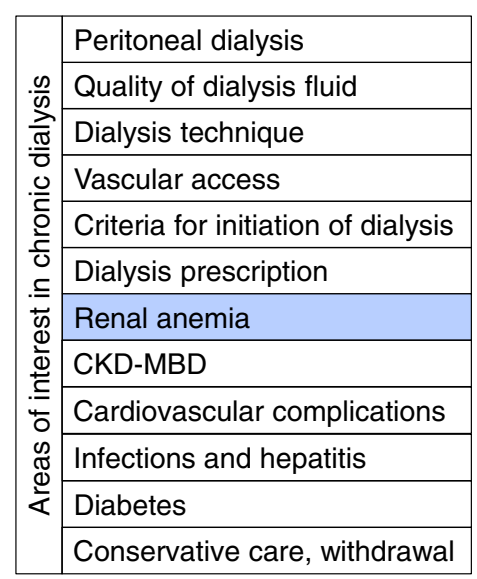

\begin{tabular}{|c|c|}
\hline \multirow{8}{*}{ 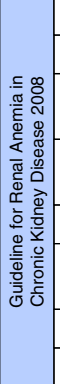 } & Chapter 1. Diagnosis, criteria, and treatment of renal anemia \\
\hline & Chapter 2. Target $\mathrm{Hb}$ level and criteria for starting ESA therapy \\
\hline & $\begin{array}{l}\text { Chapter 3. Evaluation of iron status and administration of iron } \\
\text { therapy }\end{array}$ \\
\hline & $\begin{array}{l}\text { Chapter 4. Administration of ESAs-administration } \\
\text { route/dosage }\end{array}$ \\
\hline & Chapter 5. Hypo-responsiveness (resistance) to ESAs \\
\hline & $\begin{array}{l}\text { Chapter 6. Blood transfusion in patients with chronic kidney } \\
\text { disease }\end{array}$ \\
\hline & Chapter 7. Side effects and concomitant symptoms of ESAs \\
\hline & Chapter 8. Guidelines for renal anemia in children \\
\hline
\end{tabular}

Fig. 3 Current structure of JSDT guidelines. The existing JSDT CPGs have covered almost all areas of interest in chronic dialysis (left panel) and each CPG also covered almost all topics in each area, explained with an example of 2008 JSDT Guidelines for Renal Anemia in Chronic Kidney Disease [20] (right panel). The concept of JSDT CPGs has been considered to be good for user's availability upon first reading

(Fig. 4). Under the comprehensive document, various CQs-based CPGs would be stored to a proper area of interest. Any resources which provide CPG or SR such as JSDT, Cochrane, and KDIGO could be acceptable. When existing evidence is not sufficient to develop CPGs and opinions are divided, both arguments will be provided if necessary. It should be noted that, if the convenience of users is emphasized too much, CPGs will become little more than a collection of expert opinions, just like conventional JSDT CPGs. Clear evidence must be provided when something is recommended to others. The policy described in this report was prepared to ensure that clear evidence is provided in the development of CPGs. The Academic Committee should examine the need for and the appropriate format of the comprehensive document in CPGs in the future.

\section{Conclusions}

In this report, we have reviewed the global trend of CPGs and the process of development of conventional CPGs and have reported the policy for the development of future CPGs. Conventional JSDT CPGs were user-friendly and very useful in clinical practice, having contributed to the improvement in the QOL of dialysis patients in Japan. Although the process of development of those CPGs did not fit the current global trend, many clinicians have agreed with the consensus opinion resulting from the discussion of a number of experts. However, as described herein, we should not ignore the process of development, even if we have confidence in the resulting recommendation. There is a risk that a "clinical practice guidelines" may turn out to be little more than "expert opinions" due to an inappropriate process of development. There will be an increasing

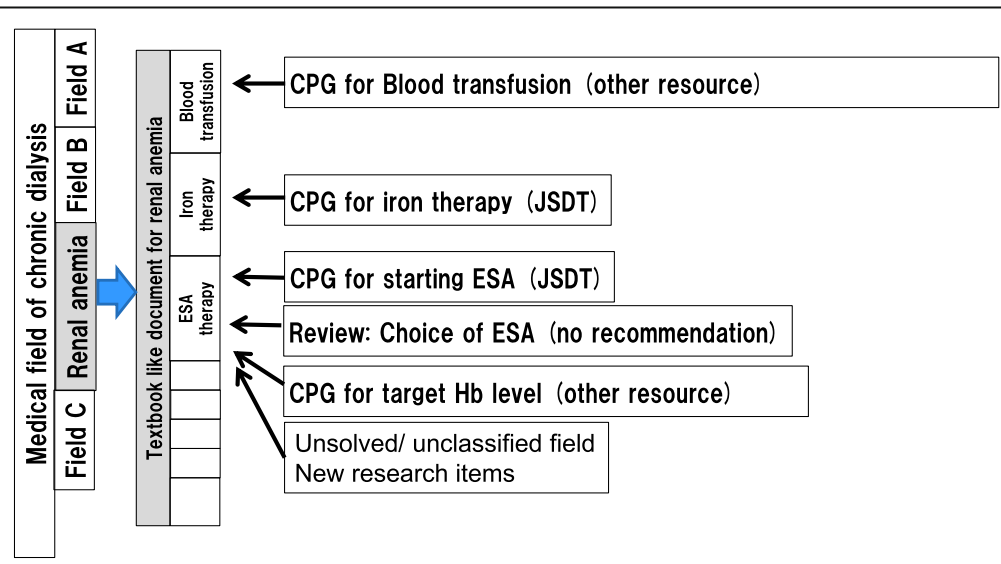

Fig. 4 Proposal for a structure of new CPG by JSDT. Under the comprehensive document various CQ-based CPGs would be stored to a proper area of interest. Any resources which provide CPG or SR could be acceptable 
need for providing the know-how of dialysis therapy accumulated in Japan to Asian countries and other regions in a globally accepted form. For this purpose, we concluded that the most reasonable approach is to utilize the data from JRDR in a more creative manner, publish such data for the world as evidence, and develop CPGs in accordance with a globally recognized methodology, GRADE.

\begin{abstract}
Abbreviations
BMJ: The British Medical Journal; CD: Committee draft; CDC: Center of Disease Control; CEBM: Centre for Evidence-Based Medicine; CKD-MBD: Chronic kidney disease-mineral and bone disorder; COI: Conflicts of interest; CPG: Clinical Practice Guideline; CQ: Clinical question; D-DPG: Draft for CPG; EBM: Evidence-based medicine; ERA/EDTA: European Renal Association/European Dialysis; FD-DPG: Final draft for CPG; GRADE: Grading of Recommendations, Assessment, Development, and Evaluation; IOM: Institute of Medicine; JRDR: Japanese Society for Dialysis Therapy, Renal Data Registry; JSDT: Japanese Society for Dialysis Therapy; KDIGO: Kidney Disease/Improving Global Outcomes; Minds: Medical Information Network Distribution System; NGC: National Guideline Clearinghouse; PICO: Patient, intervention, comparison and outcome; QOL: Quality of life; RCT: Randomized controlled trial; SR: Systematic review; Transplantation Association QOL: Quality of life; WD: Working draft; WHO: World Health Organization
\end{abstract}

\section{Acknowledgements}

We would like to express our deepest gratitude to Dr. Yuasa from the Japanese Society for Temporomandibular Joint and Dr. Aihara form the GRADE working group for kind advice for establishing the policy to develop new CPGs of JSDT.

\section{Funding}

There are no funds nor supports for the current manuscript except JSDT. Al efforts and costs for making the current report were totally given by JSDT.

\section{Availability of data and materials}

When anyone wants to use the contents from the current manuscript, they are freely available with stating "data from JSDT" and listing it in the reference section.

\section{Authors' contributions}

KN was the president of JSDT at that time and finally approved the policy to develop new CPGS of JSDT and finalized the current manuscript. IM was the chair of the subcommittee of Guidelines and finalized the contents of the current report. TT and KT were from the Academic Committee in JSDT and gave special advises to adopt the new CPG policy to the academic cycle of JSDT. KI, TO, EK, YK, YK, MT, FT, TH, NH, TH, MF, JM, HY, NW, TW, and YW were the members of subcommittee of Guidelines in JSDT, all of them collected the information about CPGs and discussed various topics to establish the policy for developing a new JSDT CPG in the current manuscript. All authors read and approved the final manuscript.

\section{Competing interests}

The authors declare that they have no competing interests.

\section{Consent for publication}

Not applicable.

\section{Ethics approval and consent to participate}

The current manuscript is a position statement of JSDT for developing new CPGs of JSDT. It does not include any data or information concerning to individual patient or person, so there are no needs of ethical approval.

\section{Publisher's Note}

Springer Nature remains neutral with regard to jurisdictional claims in published maps and institutional affiliations.

\section{Author details}

'Ex-President of Japanese Society for Dialysis Therapy, Tokyo, Japan.

${ }^{2}$ Scientific Academy Committee of Japanese Society for Dialysis Therapy,
Tokyo, Japan. ${ }^{3}$ CPG Working Group, Scientific Academy Committee of Japanese Society for Dialysis Therapy, Tokyo, Japan. ${ }^{4}$ Yabuki Hospital, 4-5-5 Shima Kita, Yamagata City, Yamagata 990-0885, Japan.

Received: 10 February 2017 Accepted: 27 April 2017

Published online: 11 May 2017

\section{References}

1. Fukagawa M, Tsukamoto Y, Tsubakihara Y, Kiazu K, Kusano E, Nakayama M, et al. Assessment of quality of evidence and strength of Recommendation for Clinical Practice Guidelines. J Jpn Soc Dial Therapy. 2010:43:347-9 (in Japanese).

2. Institute of Medicine the National Academies. Summary. In: Field M, Lohr KN, editors. Clinical Practice Guidelines: Directions for a New Program. Washington: The National Academies Press; 1990. p. 1-18. http://www.nap. edu/read/1626/chapter/2\#8.

3. Institute of Medicine the National Academies. Summary. In: Graham R, Mancher M, Wolman DM, Greenfield S, Steinberg E, editors. Clinical Practice Guidelines We Can Trust. Washington: The National Academies Press; 2011. p. 1-14. http://www.nap.edu/read/13058/chapter/2\#4. Accessed 26 Jan 2015.

4. Medical Information Network Distribution System: Minds guideline center. http://minds.jcqhc.or.jp/n/st.php. Accessed 26 Jan 2015.

5. Fukui T, Yoshida M, Yamaguchi N, editors. Minds Handbook for Clinical Practice Guideline Development 2007. Tokyo: Igaku-Shoin Ltd; 2007. in Japanese.

6. Fukui T, Yamaguchi N, editors. Minds Handbook for Clinical Practice Guideline Development 2014. Tokyo: Igaku-Shoin Ltd; 2014. in Japanese.

7. National Guideline Clearinghouse: inclusion criteria. https://www.guideline. gov/help-and-about/summaries/inclusion-criteria. Accessed 26 Jan 2015.

8. Grading of Recommendations, Assessment, Development, and Evaluation (GRADE). http://www.gradeworkinggroup.org/. Accessed 26 Jan 2015.

9. Atkins D, Best D, Briss PA, et al. GRADE Working Group: grading quality of evidence and strength of recommendations. BMJ. 2004;328:1490.

10. Aihara M. GRADE System for clinical practice guidelines. 2nd ed. Aomori: Toppan Media; 2015 (in Japanese).

11. Gejyo F, Saito A, Akizawa T, Akiba T, Sakai T, Suzuki M, et al. 2004 Japanese Society for Dialysis Therapy Guidelines for renal anemia in chronic hemodialysis patients. Ther Apher Dial. 2004:8:443-59.

12. Vanholder R, Abramowicz D, Cannata-Andia JB, Cocchi V, Cochat P, Covic A, et al. The future of European nephrology 'guidelines' - a declaration of intent by European Best Practice (ERBP). Nephrol Dial Transplant Plus. 2009;2:2013-221.

13. Uhling K, Macleod A, Craig J, Lau J, Levey AS, Levin A, et al. Grading evidence and recommendations for clinical practice guideline in nephrology: improving Global Outcome (KDIGO). Kidney Int. 2006;70:2058-65.

14. Aihara M. Japanese Clinical Practice Guidelines Using GRADE. http://www. grade-jpn.com/jp_grade/japanese_grade_cpg.html. Accessed 26 Jan 2016. (in Japanese).

15. Cochrane collaboration. http://www.cochrane.org/. Accessed 3 Feb 2016.

16. Centre for Evidence-Based Medicine: OCEBM levels of evidence. http://www. cebm.net/ocebm-levels-of-evidence/. Accessed 3 Feb 2016.

17. Cochrane collaboration: Cochrane handbook for systematic reviews of interventions. http://handbook.cochrane.org/. Accessed 3 Feb 2016.

18. Masakane I, Tomo T, Tsuchida K, Hamano T. Dialysis Therapy, 2014 Year in Review. Establishment of New Academic System in JSDT. J Jpn Soc Dial Ther. 2016:49:159-62 (in Japanese).

19. Balshem H, Helfand M, Schunemann HJ, Oxman AD, Kunz R, Brozek J, et al. GRADE guidelines: 3. Rating the quality of evidence. J Clin Epidemiol. 2011;64:401-6.

20. Tsubakihara Y, Nishi S, Akiba T, Hirakata H, Iseki K, Kubota M, et al. 2008 Japanese Society for Dialysis Therapy: guidelines for renal anemia in chronic kidney disease. Ther Apher Dial. 2010;14:240-75. 